\title{
Ansätze für eine Bifurkationsanalyse von RF LC-Tank VCOs unter Berücksichtigung nichtlinearer Bauelementegleichungen
}

\author{
C. Zorn, J.-K. Bremer, and W. Mathis \\ Leibniz Universität Hannover, Institut für Theoretische Elektrotechnik, Appelstr. 9A, 30167 Hannover, Germany
}

\begin{abstract}
Zusammenfassung. Es wird ein alternativer Ansatz zum Entwurf von vollintegrierten LC Oszillatoren mit Hilfe der Andronov-Hopf Bifurkationsanalyse unter Einbeziehung eines nichtlinearen Overall-Modells für MOS-Transistoren (EKV-Modell) vorgestellt. Das in dieser Arbeit vorgestellte Verfahren beschreibt die MOS-Kapazität des VCOs über geometrische Gundgrößen, die damit nur als Längen- und Weitenverhältnisse in die Bifurkationsanalyse eingehen. Zur Beschreibung der MOS-Kapazität wurde ein Basic-ChargeModell, wie es in den Arbeiten von Enz und Vittoz vorgestellt wurde, in Verbindung einer expliziten analytischen Näherung des Oberflächenpotenzials verwendet. Das Verfahren ermöglicht es, als zusätzlichen Freiheitsgrad für den Designer auch die Amplitude zur Optimierung der Bauteilparameter heranzuziehen und vorab eine genauere Abschätzung der Parameter des Varaktors zu erhalten. Zusammengefasst in einer Toolbox führt die Anwendung des Verfahrens auf einen grafischen Optimierungsprozess, mit dessen Hilfe die Parameter analytisch bestimmt werden können. Die verwendete Methode erweitert den von Hajimiri und Ham vorgestellten Entwurfsprozess von LC-Tank VCOs um eine Stabilitätsanalyse, die Berücksichtigung harmonischer höherer Ordnung und die physikalisch exakte Modellierung der Varaktorkapazitäten.
\end{abstract}

\section{Einleitung}

Die Oszillatorschaltung bzw. die spannungsgesteuerte Oszillatorschaltung ist heute eine der wichtigsten integrierten Schaltungen. Sie findet in fast allen Anwendungsbereichen der mobilen Kommunikationstechnik, wie Bluetooth, WIMAX, WLAN, UMTS und GSM Anwendung und ist zentrales Thema vieler aktueller Forschungsarbeiten. Die da-

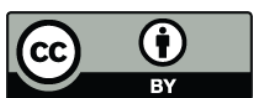

Correspondence to: J.-K. Bremer (bremer@tet.uni-hannover.de) bei behandelten Probleme, wie die Forderungen nach geringem Leistungsbedarf, geringem Phasenrauschen, einem hohen Integrationsgrad und geringen Kosten bei der Herstellung, ergeben sich zum größten Teil durch die hohe Mobilität der Endgeräte, in denen integrierte Oszillatorschaltungen zum Einsatz kommen. Der Entwurf von Oszillatoren ist dementsprechend eine sehr komplexe Aufgabe und bedarf einer ständigen Optimierung der dazu angewandten Entwurfsmethoden. Demzufolge muss für einen verbesserten Entwurfsprozess ein stärkeres Verständnis der physikalischen Vorgänge im Oszillators entwickelt und diese mit Hilfe von mathematisch verbesserten Modellen beschrieben werden. Da der Oszillator ein nichtlineares System ist, kann eine genauere Beschreibung und Analyse des Oszillators nur auf einer nichtlinearen Betrachtungsweise basieren. Aus diesem Grund müssen bei Oszillatoren lineare Beschreibungen durch nichtlineare Beschreibungen erweitert oder auch ersetzt werden. Wir zeigen eine Erweiterung der nichtlinearen Modellierung des Oszillators mit Hilfe der AndronovHopf-Bifurkation, wie sie schon in vorangegangenen Arbeiten durchgeführt wurde (Mathis, 2004, 1987; Prochaska et al., 2005). In einem neuartigen Ansatz werden die Beschreibungsgleichungen von Oszillatoren durch Hinzufügen einer spannungsabhängigen Kapazität erweitert, so dass man einen spannungsgesteuerten Oszillator erhält, auf den eine Bifurkationsanalyse angewendet werden kann. Die dafür gewählte Schaltungstopologie ist der LC-Tank Oszillator, da dieser wegen seiner guten Phasenrauscheigenschaften und einem hohen Integrationsgrad beim Entwurf von integrierten analogen Schaltungen sehr weit verbreitet ist.

\section{Andronov-Hopf Bifurkation}

Den Ausgangspunkt der Andronov-Hopf Bifurkation bildet ein nichtlineares dynamisches System folgender Form:

$\dot{\mathbf{x}}=\mathbf{f}(\mathbf{x}, \mu), \mathbf{f}: \mathbb{R}^{n} \rightarrow \mathbb{R}^{n}, \mathbf{x} \in \mathbb{R}^{n}$. 


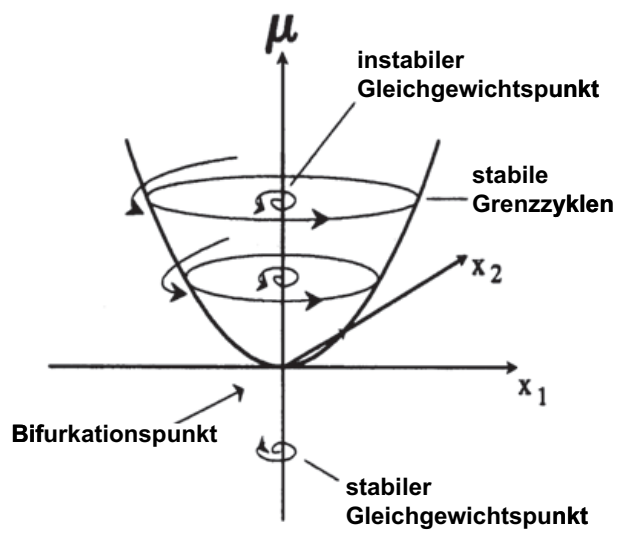

Abbildung 1. Andronov-Hopf Bifurkation.

In der Funktion $\mathbf{f}(\mathbf{x}, \mu)$ stellt $\mu$ einen freien Netzwerkparameter oder eine Kombination aus Parametern dar, die zur späteren Optimierung zur Verfügung stehen. Zunächst müssen die Art und die sich daraus ergebenen Zustände des Systems betrachtet werden. Ist der Parameter $\mu$ sinnvoll gewählt erwartet man für $\mu<0$, dass sich das System in einem stabilen Arbeitspunkt befindet und dieser dann für $\mu>0$ in einen stabilen Grenzzyklus übergeht. Dies entspricht dem eingeschwungenen Zustand eines elektrischen Oszillators.

An dieser Stelle wird die Andronov-Hopf Bifurkation zur Beschreibung des Systems genutzt, da die eben genannten Erwartungen genau dann erfüllt werden, wenn die Voraussetzungen dieses Theorems erfüllt sind.

Satz von Andronov-Hopf

Sind die folgenden Voraussetzungen

1. Die Jacobi-Matrix $\left.\mathbf{J}_{f}(\mathbf{x}, \mu)\right|_{\mu=0} ^{x=0}$ besitzt ein konjugiert komplexes Eigenwertpaar $\lambda_{1,2}= \pm j \omega_{0}$

2. Alle weiteren Eigenwerte haben einen negativen Realteil

3. Die Transversalitätsbedingung $\left.\frac{d}{d \mu} \Re \lambda(\mu)\right|_{\mu=0}>0$ ist erfüllt

4. Der Gleichgewichtspunkt ist für $\mu=0$ asymptotisch stabil

erfüllt, dann existieren zwei hinreichend kleine Parameter $\mu_{1}$ und $\mu_{2}$, so dass im Intervall $\left(-\mu_{1}, 0\right)$ alle transienten Lösungen spiralförmig in den stabilen Gleichgewichtspunkt $\mathbf{0}$ einlaufen. Für das Intervall $\left(0, \mu_{2}\right)$ existiert als Lösung des Systems eine isolierte geschlossenen Kurve im Zustandsraum, die im Rahmen der mathematischen Theorie dynamischer Systeme Grenzzyklus genannt wird. Alle transienten Lösungen mit Anfangsbedingungen die nicht auf dem Grenzzyklus starten laufen asymptotisch in den Grenzzyklus ein. Eine Vergrößerung des Bifurkationsparameters führt zu einer

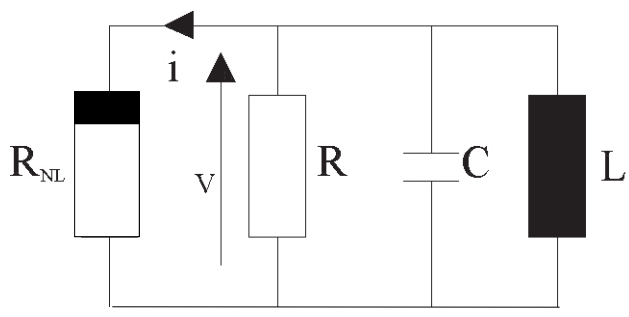

Abbildung 2. Vereinfachtes Ersatzschaltbild.

Aufweitung des Grenzzyklus und dementsprechend zu einem Anstieg der Oszillationsamplitude (vgl. Guckenheimer und Holmes, 1983, und Mathis und Russer, 2005).

\section{Bifurkationsanalyse von LC-Tank Oszillatoren}

Um die Bifurkationsanalyse im Sinne früherer Arbeiten (z.B. Prochaska et al., 2005) auf den Designprozess von Oszillatoren anwenden zu können, wird zunächst das allgemeine Kleinsignalersatzschaltbild eines LC-Tank Oszillators, wie es von Hajimiri (Ham und Hajimiri, 2001) bekannt ist, weiter vereinfacht. Das Ersatzschaltbild wird für unsere Zwecke auf eine Parallelschaltung aus Induktivität, Kapazität, den in einem ohmschen Widerstand zusammengefassten Verlusten und einem nichtlinearen Widerstand zur Kompensation der Verluste zurückgeführt. Dafür müssen aus dem Ersatzschaltbild nach Hajimiri zunächst alle Reihenschaltungen in Parallelschaltungen umgerechnet werden (siehe Abb. 2). Ausgehend von dem zuvor gezeigten Ersatzschaltbild, wird nun ein nichtlineares dynamisches System zweiter Ordnung mit Hilfe von Netzwerkgleichungen aufgestellt. Dabei steht der Index $t$ für die Schwingkreisgrößen (engl. Tank).

Die Spannung über der Induktivität ist beschrieben mit

$V_{t}=L_{t} \frac{d i_{L}}{d t}$

Der Strom durch den nichtlinearen Widerstand wird über die Summe der Ströme im Knoten berechnet

$-i_{d}\left(V_{t}\right)=i_{R}+i_{C}+i_{L}$

$i_{d}\left(V_{t}\right)=\frac{V_{t}}{R_{t}}+C_{t} \frac{d V_{t}}{d t}+i_{L}$.

Aus der Spannung und der Summe der Knotenströme ergeben sich folgende Differenzialgleichungen

$\frac{d V_{t}}{d t}=-\frac{V_{t}}{R_{t} C_{t}}-\frac{i_{L}}{C_{t}}-\frac{i_{d}\left(V_{t}\right)}{C_{t}}$ 
$\frac{d i_{L}}{d t}=\frac{V_{t}}{L_{t}}$

Diese bilden zusammengefasst folgendes nichtlineare dynamische System zweiter Ordnung

$$
\left(\begin{array}{l}
\frac{d V_{t}}{d t} \\
\frac{d i_{L}}{d t}
\end{array}\right)=\left(\begin{array}{cc}
-\frac{1}{R_{t} C_{t}} & -\frac{1}{C_{t}} \\
\frac{1}{L_{t}} & 0
\end{array}\right)\left(\begin{array}{l}
V_{t} \\
i_{L}
\end{array}\right)-\left(\begin{array}{c}
\frac{i_{d}\left(V_{t}\right)}{C_{t}} \\
0
\end{array}\right),
$$

was einem Differenzialsystem der Form

$\dot{\mathbf{x}}=\mathbf{f}(\mathbf{x}, \mu), \mathbf{f}: \mathbb{R}^{n} \rightarrow \mathbb{R}^{n}, \mathbf{x} \in \mathbb{R}^{n}$.

entspricht. Für die Beschreibung des von der Oszillatoramplitude abhängigen Stromes $i_{d}$ durch den nichtlinearen Widerstand wurde ein von Buonomo (Buonomo und Lo Schiavo, 2002) vorgestellter Ansätze gewählt,

$i_{d}\left(V_{t}\right)=-I_{\mathrm{Bias}} \frac{V_{t}}{v_{n}} \sqrt{1-\frac{V_{t}^{2}}{v_{n}^{2}}}$

mit

$$
v_{n}=2 \sqrt{\frac{I_{\mathrm{Bias}} L_{n}}{\mu_{n} C_{o x} W_{n}}} .
$$

Die Größen $W_{n}$ und $L_{n}$ sind Weite und Länge der Transistoren, die zur Realisierung des nichtlinearen Widerstandes verwendet werden. Da der VCO über die Änderung der Kapazität in seiner Frequenz abgestimmt wird, dass oben gezeigte System jedoch nur eine Beschreibung mit konstanten Kapazität ist, beziehen sich alle Berechnungen nur auf den Oszillator mit fester Frequenz. Zur Beschreibung eines spannungsgesteuerten Oszillators muss der Ansatz zur Berechnung der Amplitude um ein nichtlineares Modell der Kapazität erweitert werden. Dabei soll angemerkt werden, dass auch schon im Falle eines Oszillators mit fester Frequenz, auf Grund der Dynamik des Systems, nicht von einer konstanten Kapazität ausgegangen werden kann. Somit ergibt sich das neue erweiterte Ersatzschaltbild (siehe Abb. 4).

\section{Oberflächenpotenzial basierte Modellierung von MOS-Kapazitäten}

Um das System der zuvor erläuterten Bifurkationsanalyse auf den VCO zu erweitern, wird eine analytische Beschreibung der verwendeten MOS-Kapazität benötigt. In den meisten Modellen (z.B. aus der BSIM-Familie) werden dazu die einzelnen Arbeitsbereiche eines MOS-Transistors durch Näherungen beschrieben und am Ende zu einer Gesamtbeschreibung zusammengefügt. Beim Basic Charge Modell (Enz und Vittoz, 2006) handelt es sich um ein so genanntes Overall-Modell, welches eine kontinuierliche Beschreibung der MOS-Kapazität in allen Bereichen liefert. Die

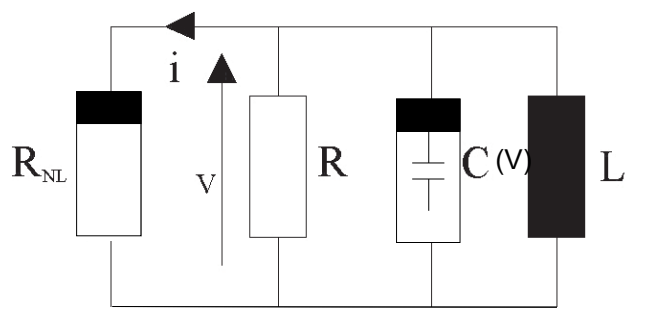

Abbildung 3. Um eine spannungsabhängige Kapazität erweitertes Ersatzschaltbild.

grundlegende Idee dabei ist, eine Betrachtung des MOSVaraktorverhaltens in Abhängigkeit der Ladungsverteilung bzw. des Oberflächenpotenzials des Halbleiters anzustellen. Dabei wird die vom Gleichgewicht abweichende Ladungsverteilung im Inneren des Halbleiters berechnet und daraus die unter der Oberfläche befindliche Ladungsflächendichte bestimmt. Im Folgenden werden alle Berechnungen auf ein p-dotiertes Substrat bezogen und zur Vereinfachung wird davon ausgegangen, dass alle Dotieratome (Akzeptoren) im Substrat ionisiert sind (Störstellenerschöpfung). Die Gesamtkapazität

$C_{\mathrm{ges}}=\frac{C_{s i} \cdot C_{o x}}{C_{s i}+C_{o x}}$,

berechnet sich dabei aus der Reihenschaltung der konstanten Oxidkapazität

$C_{o x}=\frac{\epsilon_{o x} * \epsilon_{0} * A}{t_{o x}}$

und der aus dem Zusammenhang

$C_{s i}=\frac{d\left(-Q_{s i}\right)}{d \psi_{s}}$

abgeleiteten spannungsabhängige Kapazität über der Raumladungszone

$$
\begin{aligned}
C_{s i} & =\frac{\epsilon_{s i} U_{T}}{L_{D}} \frac{d F}{d \psi_{s}} \\
& =\frac{\epsilon_{s i}}{2 L_{D}} \frac{\exp \left(\frac{\psi_{s}-2 \phi_{F}-V}{U_{T}}\right)-\exp \left(\frac{-\psi_{s}}{U_{T}}\right)+1}{F\left(\psi_{s}, 2 \phi_{F}+V\right)} .
\end{aligned}
$$

Da es sich bei der Gleichung um die Abhängigkeit der Kapazität vom Oberflächenpotenzial handelt, man das Oberflächenpotenzial jedoch nicht als einstellbaren Parameter zur Verfügung hat, benötigt man eine Abhängigkeit der Kapazität von der Gatespannung. Der Zusammenhang zwischen $\psi_{s}$ und $V_{G}$

$\frac{V_{G}-V_{F B}}{U_{T}}=\frac{\psi_{s}}{U_{T}}+\frac{\epsilon_{s i}}{C_{o x} L_{D}} F\left(\psi_{s}, 2 \phi_{F}+V\right)$ 


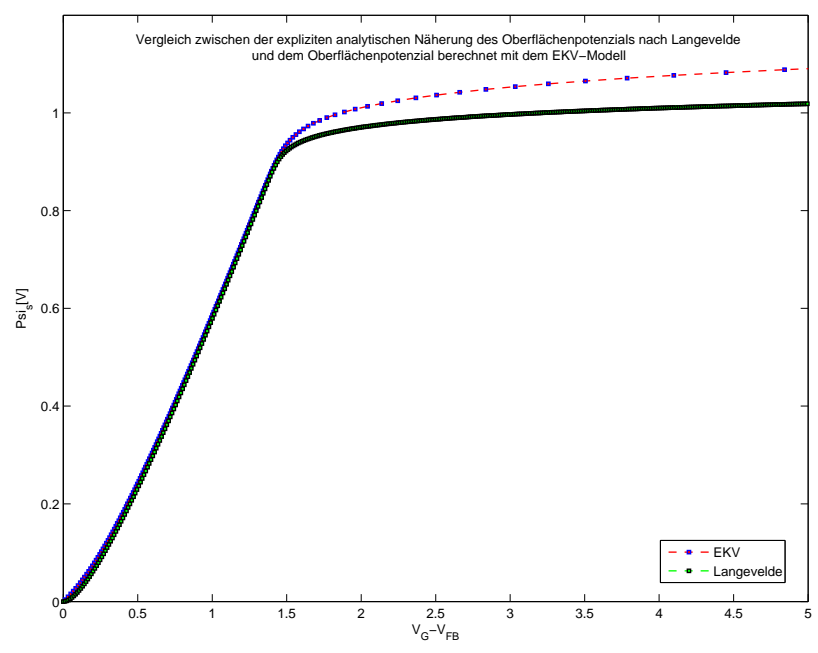

Abbildung 4. Vergleich zwischen der expliziten analytischen Näherung des Oberflächenpotenzials nach Langevelde und dem mit dem EKV-Modell berechneten Oberflächenpotenzial.

( $V_{G}$ ist die Gatespannung, $\psi_{s}$ ist das Oberflächenpotenzial, $t_{o x}$ die Dicke des Oxides und $\phi_{m s}$ ein Potentialunterschied zwischen Gate und Kanalmaterial) ist jedoch nicht invertierbar, es handelt sich um einen impliziten Ausdruck. Zur expliziten analytischen Darstellung des Oberflächenpotenzials sind in der Vergangenheit unterschiedlichste Arbeiten veröffentlicht worden (Chen und Gildenblatt, 2001; van Langevelde und Klaassen, 2000). Da der von Langevelde vorgestellte Ansatz einen guten Kompromiss zwischen Rechenaufwand und Genauigkeit bietet, wurde dieser von uns dazu verwendet, dass für die Bifurkationsanalyse notwendige System aufzustellen. Der Ausdruck für die explizite analytische Näherung des Oberflächenpotenzials von Langevelde beschränkt sich auf den Bereich von Depletion bis Inversion. Das bedeutet für unseren Anwendungsfall keine Einschränkung, da verwendeten Varaktoren nur in diesem Bereich arbeiten. Mit den zusätzlichen Annahmen, $\psi>0$ und $\psi \gg u_{t}$, kann die Gl. (14) $\mathrm{zu}$

$V_{G}-V_{F B}-\psi=\gamma \sqrt{\psi+u_{t} \cdot \exp \left(\frac{\psi-V-2 \phi_{F}}{u_{t}}\right)}$

reduziert werden. Die Temperaturspannung $u_{t}$ ist definiert als $k T / q$ und der Faktor $\gamma$ entspricht $\sqrt{2 q \varepsilon_{s i} N_{a}} / C_{o x}$. Um aus dem vereinfachten Ausdruck eine explizite Darstellung zu entwickeln, müssen einige Näherungen aufgestellt werden. Für den Bereich der schwachen Inversion

$0<\psi<2 \phi_{F}+V$

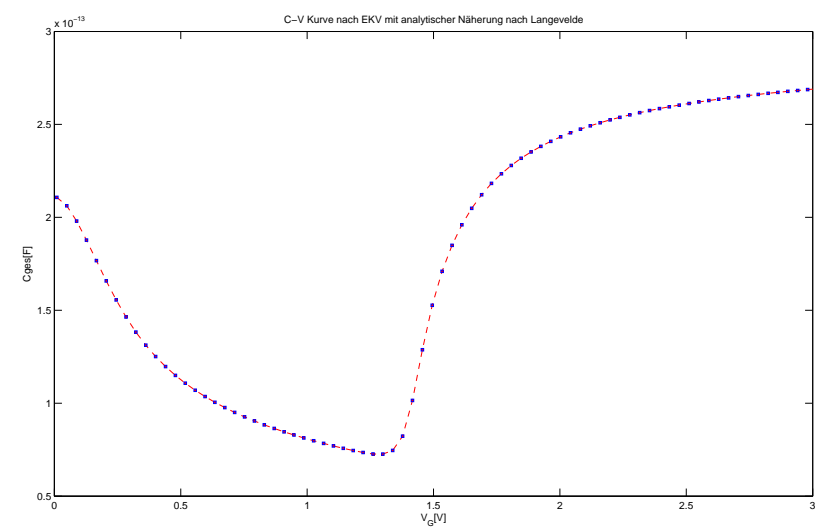

Abbildung 5. Gesamtkapazität $\left(C_{\text {ges }}\right)$ in Abhängigkeit der Gatespannung für $V=0$ mit analytischer Näherung.

lässt sich Gl. (15) unter Vernachlässigung des exponentiellen Anteils, wie folgt bestimmen

$\psi_{w i}=\left(\sqrt{V_{G B}-V_{F B}+\frac{\gamma^{2}}{4}-\frac{\gamma}{2}}\right)^{2}$.

Um letztendlich ein kontinuierlichen Übergang vom Bereich der starken Inversion zum Bereich der schwachen Inversion zu erreichen nutzt Langevelde die folgende SmoothingFunction (Velghe et al., 1994)

$$
\begin{aligned}
f\left(V_{G B}, V\right)= & \frac{2 \phi_{F}+V}{2}+\frac{\psi_{w i}}{2} \\
& -\frac{1}{2} \sqrt{\left(\psi_{w i}-2 \phi_{F}-V\right)^{2}+4 \epsilon^{2}} .
\end{aligned}
$$

Dabei ist $\epsilon$ ein Anpassungsfaktor mit dem Wert 2e-2. Nach einigen Umformungen erhält man die endgültige explizite Näherung des Oberflächenpotenzials

$$
\begin{gathered}
\psi=f+u_{t} \ln \left[\left(\frac{V_{G B}-V_{F B}-f}{\gamma \sqrt{u_{t}}}\right.\right. \\
\left.\left.-\frac{\left(\psi_{w i}-f\right) \cdot \gamma \sqrt{u_{t}}}{\sqrt{1+\left[\frac{\psi_{w i}-f}{4 u_{t}}\right]^{2}}}\right)^{2}-\frac{f}{u_{t}}+1\right]
\end{gathered}
$$

Abbildung 4 zeigt die mit unserer symbolischen Umsetzung erzeugte analytische Näherung des Oberflächenpotenzials im Vergleich zur impliziten Berechnung mit dem EKV Modell.

Die Näherung des Oberflächenpotenzials von Langevelde ist für die Bifurkationsanalyse geeignet und liefert wie in Abb. 4 gezeigt ein genaues Ergebnis. Die aus dem EKV Modell, in Kombination mit der expliziten analytischen Näherung, entstehenden Gesamtkapazität in Abhängigkeit der Gatespannung ist in Abb. 5 zu sehen. 


\section{Bifurkationsanalyse mit veränderlicher Kapazität}

Für die Bifurkationsanalyse mit veränderlicher Kapazität wird das nichtlineare dynamische System (6) erweitert. Dafür wird das vorgestellte Surface Potential Modell in Kombination mit der expliziten analytischen Näherung für das Oberflächenpotenzial nach Langevelde genutzt. Alle Größen sind dabei auf geometrische Grundgrößen zurückgeführt und gehen somit nur als Längen und Weiten in die Bifurkationsanalyse ein. Das somit entstehende neue Gesamtsystem lautet wie folgt:

$$
\begin{aligned}
& \left(\begin{array}{c}
\frac{d V_{t}}{d t} \\
\frac{d i_{L}}{d t}
\end{array}\right)=\left(\begin{array}{cc}
{\left[-\frac{1}{a_{0} W L R_{t}}+\frac{I_{\text {Bias }}}{a_{0} W L v_{n}}\right.} & {\left[-\frac{1}{a_{0} \cdot W L}\right]} \\
0 & 1 / L_{t}
\end{array}\right) \\
& \times \cdot\left(\begin{array}{c}
V_{t} \\
i_{L}
\end{array}\right)+\left(\frac{1}{W L}\left[\begin{array}{c}
A_{1} V_{t}^{2}+A_{2} V_{t}^{3}+A_{3} V_{t}^{4} \\
+A_{4} V_{t}^{5}+A_{5} V_{t}^{6}+A_{6} V_{t} i_{L} \\
+A_{7} V_{t}^{2} i_{L}+A_{8} V_{t}^{3} i_{L} \\
0
\end{array}\right]\right)
\end{aligned}
$$

mit

$$
\begin{aligned}
& A_{1}=\left[-\frac{1}{a_{1} R_{t}}+\frac{I_{\mathrm{Bias}}}{a_{1} v_{n}}\right] \\
& A_{2}=\left[-\frac{1}{a_{2} R_{t}}+\frac{I_{\mathrm{Bias}}}{a_{2} v_{n}}-\frac{I_{\mathrm{Bias}}}{a_{0} 6 v_{n}^{3}}\right] \\
& A_{3}=\left[-\frac{1}{a_{3} R_{t}}+\frac{I_{\mathrm{Bias}}}{a_{3} v_{n}}-\frac{I_{\mathrm{Bias}}}{a_{1} 6 v_{n}^{3}}\right], A_{4}=\left[-\frac{I_{\mathrm{Bias}}}{a_{2} 6 v_{n}^{3}}\right] \\
& A_{5}=\left[-\frac{I_{\mathrm{Bias}}}{a_{3} 6 v_{n}^{3}}\right], A_{6}=-\frac{1}{a_{1}}, A_{7}=-\frac{1}{a_{2}}, A_{8}-\frac{1}{a_{3}}
\end{aligned}
$$

Dies entspricht einem Differenzialsystem der Form

$\dot{\mathbf{x}}=\mathbf{A x}+\tilde{\mathbf{f}}(\mathbf{x})$

wobei $\tilde{\mathbf{f}}(\mathbf{x})$ den nichtlinearen Anteil repräsentiert.

Nach Zerlegung des nichtlinearen Systems in einen linearen und nichtlinearen Anteil kann die Andronov-Hopf Bifurkationsanalyse angewendet werden. Sind alle Kriterien des Andronov-Hopf Theorems erfüllt, ist sichergestellt, dass ein Bifurkationsparameter $\mu$ existiert, dessen Variation zu einem stabilen Grenzzyklus führt. Im ersten Schritt muss der Bifurkationsparameter im System ausgewählt werden. Der Parameter muss die Einstellung des Bifurkationspunktes ermöglichen und darf darüber hinaus die Oszillationsfrequenz nicht beeinflussen. In dem Gleichungssystem (20) ist daher die Weite des Transistorpaars $W_{n}$ ein geeigneter Bifurkationsparameter, da diese im Vergleich zur Weite des Varaktors $W$ die Frequenz des Oszillators wenig beeinflusst. Die erste Voraussetzung für das Andronov-Hopf Theorem besagt, dass das li- nearisierte System (20) im Bifurkationspunkt ein konjugiertkomplexes Eigenwertpaar mit verschwindendem Realteil besitzt. Die Eigenwerte der Systemmatrix lassen sich aus

$\operatorname{det}(\mathbf{A}-\lambda \mathbf{E})=\left[\begin{array}{cc}(\alpha-\lambda) & -\frac{1}{a_{0} W L} \\ \frac{1}{L_{t}} & -\lambda\end{array}\right]=0$

wie folgt bestimmen:

$\lambda_{1,2}=+\frac{\alpha}{2} \pm \sqrt{\left(\frac{\alpha}{2}\right)^{2}-\frac{1}{a_{0} W L L_{t}}}$,

mit

$\alpha=-\frac{1}{a_{0}}\left[-\frac{1}{\mathrm{WLR}_{t}}+\frac{I_{\mathrm{Bias}}}{W L \mu_{n}}\right]$.

Im Bifurkationspunkt muss nach der Beziehung (23) der Parameter $W_{n}$, von dem $\alpha$ abhängig ist, so eingestellt werden, dass die Bedingung $\alpha=0$ eingehalten wird. Dann ergeben sich die Eigenwerte $\mathrm{zu}$

$\lambda_{1,2}= \pm j \sqrt{\frac{1}{a_{0} W L L_{t}}}$.

Daraus lässt sich die Oszillationsfrequenz direkt bestimmen

$\omega_{0}=\sqrt{\frac{1}{a_{0} W L L_{t}}}$.

Da der Parameter $a_{0}$ von der Tuningspannung $V_{\text {tune }}$ abhängig ist, kann man die Oszillationsfrequenz durch Variation von $V_{\text {tune }}$ abstimmen, ohne dass sich die Bifurkationsbedingung $\alpha=0$ ändert. Dies folgt offensichtlich aus Gl. (25). Damit können zunächst einige notwendige Bedingungen (vgl. Barkhausen-Kriterium) des Andronov-Hopf Theorems erfüllt werden.

Es sei angemerkt, dass eine lineare Abhängigkeit der Oszillationsfrequenz $f_{0}\left(\omega_{0}=2 \pi f_{0}\right)$ von der Tuningspannung $V_{\text {tune }}$ nur dann gegeben ist, wenn $a_{0}\left(V_{\text {tune }}\right)$ umgekehrt proportional $\mathrm{zu} V_{\text {tune }}^{2}$ abhängt. In den Anwendungen ist dies durch geeignete schaltungstechnische Maßnahmen sicherzustellen.

\section{Zusammenfassung}

Um die nichtlineare Kapazität in der Bifurkationsanalyse nutzen zu können, musste für die vom Oberflächenpotenzial abhängigen Kapazität ein Ausdruck in Abhängigkeit der Gatespannung entwickelt werden. Da es sich bei dem Zusammenhang zwischen Oberflächenpotenzial und Gatespannung um eine implizite Funktion handelt, wurde diese mit Hilfe einer Näherung in expliziter Weise beschrieben. Zu diesem Zweck wurden die Ansätze zur expliziten Näherung des Oberflächenpotenzials von Gildenblat (Chen 
und Gildenblatt, 2001) und Langevelde (van Langevelde und Klaassen, 2000) unter Matlab implementiert und mit der impliziten Lösung des Oberflächenpotenzials verglichen. Das in dieser Arbeit vorgestellte Modell beschreibt die MOSKapazität in einem VCO über geometrische Grundgrößen und geht damit nur als Längen- und Weitenverhältnisse in die Bifurkationsanalyse ein. Damit wurde erstmalig nichtlineare Beschreibungsgleichungen für VCOs mit spannungsabhängiger Kapazität formuliert, die als Ausgangspunkt für eine Bifurkationsanalyse geeignet sind. Einige notwendige Bedingungen konnten bereits in dieser Arbeit erfüllt werden. Auf dieser Grundlage soll in folgenden Arbeiten eine Entwurfsmethodik für VCOs entwickelt werden, welche die Nichtlinearitäten von VCOs in umfassender Weise einbezieht. Die vollständige Bifurkationsanalyse ist dabei in gleicher Weise durchzuführen, wie das in früheren Arbeiten für LC-Tank Oszillatoren bereits geschehen ist; vgl. Prochaska et al. (2005).

\section{Literatur}

Buonomo, A. und Lo Schiavo, A.: Analyzing the Dynamic Behavior of RF Oscillators IEEE Trans. Circuits and Systems, 49(11), 2002.

Chen, T. L. und Gildenblat, G.: Analytical approximation for the MOSFET surface potebtial, Solid-State Electronics, 45, 2001.

Enz, C. C. und Vittoz, E. A.: Charge-based MOS Transistor Modeling, John Wiley and Sons, 2006.
Grabinski, W., Nauwelaers, B., und Schreurs, D.: Transistor level Modeling for Analog/RF IC Design, Springer Verlag, 2006.

Guckenheimer, J. und Holmes, P.: Nonlineare Oscillations, Dynamical Systems, and Bifurcations of Vector Fields, Springer Verlag, 2006.

Ham, D. und Hajimiri, A.: Concepts and Methods in Optimization of Integrated LC VCOs, IEEE J. Solid-State Circuits, 36(6), 2001.

Mathis, W.: Theorie nichtlinearer Netzwerke, Springer Verlag, Berlin, 1987.

Mathis, W.: Bifurcations in Noisy Nonlinear Networks and Systems, in: Complex Computing-Networks, herausgegeben von: Göknar, I. C. und Sevgi, L., Brainlike and Wave-oriented Electrodynamic Algorithms, Springer Proceedings in Physics, SpringerVerlag, Berlin - Heidelberg, 2006.

Mathis, W. und Russer, P.: Oscillator Design, in: Encyclopedia of RF and Microwave Engeneering Chang, K. (Herausg.), 4, 35633589, Wiley, 2005.

Prochaska, M., Belski, A., und Mathis, W.: Bifurcation analysis of on-chip LC VCOs, IEEE International Symposium on Circuits and Systems ISCAS, 6, 5445-5448, 2005.

van Langenvelde, R. und Klaassen, F. M.: An explicit surface potebtial-based MOSFET model for circuit simulation SolidState Electronics, 44, 2000.

Velghe, R. M., Klaassen, D. B., and Klaassen, F. M.: Unclassified report NL-UR 003/94: MOS model 9, Eindhoven: Philips Electronics, 1994. 